\title{
A Novel Model for Predicting Sun Outage in Satellite Communication
}

\author{
Lihua $\mathrm{Ma}^{1}$, Chao $\mathrm{Hu}^{1}$, Jun $\mathrm{Pei}^{1}$, Xinghua $\mathrm{Ma}^{1} \&$ Yanben $\mathrm{Han}^{1}$ \\ ${ }^{1}$ National Astronomical Observatories, Chinese Academy of Sciences, Beijing, China \\ Correspondence: Lihua Ma, National Astronomical Observatories, Chinese Academy of Sciences, Beijing \\ 100012, China. Tel: 86-10-64807751.E-mail: mlh@nao.cas.cn
}

Received: December 27, 2017

Accepted: January 10, 2018 Online Published: January 18, 2018

doi:10.5539/esr.v7n1p106

URL: https://doi.org/10.5539/esr.v7n1p106

The research is financed by the National Natural Science Foundation of China (Grant No. 11573041, 11473045).

\begin{abstract}
A sun outage is an interruption or distortion of satellite communication signals caused by interference from solar radiation. There is a serious interference to satellite communication services in a special period of time. In the present paper, a novel model for predicting sun outage that is effective and can greatly improve accuracy and precision of sun outage prediction of earth station in satellite communication is presented.
\end{abstract}

Keywords: satellite communication, earth station, sun outage

\section{Introduction}

Communication satellites locate at the altitude with tens of thousands of kilometers above the surface of the Earth, so the satellite signals to the ground are very weak. The Sun radiates strongly across the entire spectrum, including the microwave frequencies (such as $\mathrm{C}$-, $\mathrm{Ku}$ - and Ka-band) used to communicate with satellites, and to send high levels of electromagnetic radiation to the satellite. It will result in an interruption in or distortion of satellite signals. It is called a sun outage, also called as sun transit in astronomy. The effects of a sun outage range from partial degradation (increase in the error rate) to total destruction of the signals (Note 1). When a strong interference electromagnetic signal from the Sun and the satellite signals are in the same direction, the Sun's wide electromagnetic spectrum is a huge noise source for receiving antenna on the earth station. The communication signals are overwhelmed by the Sun's radiation, and the demodulation system can't distinguish between the satellite signals and the Sun's interference signal (Song et al. 2010; Guo et al., 2012; Lou et al., 2016).

Communication satellites are often placed in the geostationary Earth orbit (GEO), so that the satellite antennas on the earth station that communicate with them do not have to rotate to track them, but can be pointed permanently at the position in the sky where the satellites are located (Pratt et al., 2003). Considering the motion characteristics of the Sun, as for GEO communication satellites, in the northern hemisphere, sun outages occur before the March equinox (February and March) and after the September equinox (September and October), and in the southern hemisphere the outages occur after the March equinox and before the September equinox. At these times, the apparent path of the Sun across the sky takes it directly behind the line of sight between the earth station and the GEO satellite. During the solar interference, to calculate the time of solar interference, the precise position of the Sun is necessary. Based on the Swiss ephemeris, a novel model for predicting the sun outage is proposed in this work.

\section{Swiss Ephemeris}

The Swiss ephemeris is the high precision ephemeris developed by Astrodienst (Note 2), largely based upon the DExxx ephemerides from NASA's Jet Propulsion Laboratory (JPL). The original release in 1997 was based on the DE405/406 ephemeris. Since release 2.00 in February 2014, it is based on the DE431 ephemeris released by JPL in September 2013. The Swiss ephemeris covers the time range 13201 BC to AD 17191. These data have been further compressed with sophisticated compression techniques developed by Astrodienst. This compressed ephemeris reproduces the JPL data with 0.001 arcseconds precision. All transformation steps from the inertial timeframe of the JPL DE430/431 integration to the reference frame for astrological coordinates (true equinox of date), all corrections like relativistic aberration, deflection of light in the gravity field of the Sun etc. have been 
performed with utmost care and precision so that the target precision of 0.001 arcseconds is maintained through all transformation steps.

The Swiss ephemeris provides a function package for the computation of planetary positions (Tian et al., 2007). It includes the planets, the moon, the lunar nodes, the lunar apogees, the main asteroids, Chiron, Pholus, the fixed stars and several "hypothetical" bodies. Hundreds of other minor planets are included as well. The Swiss ephemeris package consists of a DLL, a collection of ephemeris files and a few sample programs which demonstrate the use of the DLL and the Swiss Ephemeris graphical label. In addition, full C source codes are included with the Swiss Ephemeris, so that programmers also can create a linkable or shared library in their environment and use it with their applications.

\section{Novel Model for Predicting Sun Outage}

\subsection{Occurrence Condition of Sun Outage}

Firstly, a geocentric celestial reference system is established. Here the location of the communication antenna is as the coordinate origin. Figure 1 gives a diagrammatic sketch of the geocentric celestial reference system. The Point $Z$ is the zenith. The communication satellite and the Sun are projected at point $R$ and $S$, respectively. Their spherical coordinates are $\left(A_{R}, E_{R}\right)$ and $\left(A_{S}, E_{S}\right)$, respectively.

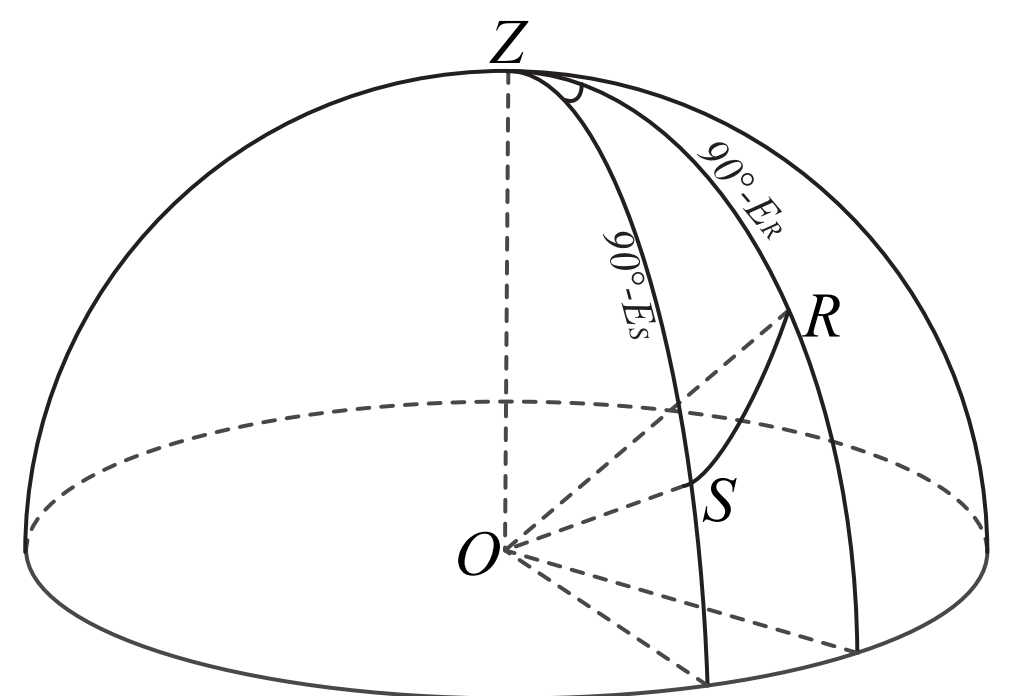

Figure 1. Diagrammatic sketch of the geocentric celestial reference system

In the spherical triangle $\triangle Z R S$, according to the spherical law of cosines, the following equation is obtained.

$$
\cos \theta=\cos \left(90^{\circ}-E_{R}\right) \cos \left(90^{\circ}-E_{S}\right)+\sin \left(90^{\circ}-E_{R}\right) \sin \left(90^{\circ}-E_{S}\right) \cos \left(A_{S}-A_{R}\right)
$$

Where, the angle $\theta$ is an included angle of point $R$ and point $S$ on the spherical surface.

On the earth station, half-power beam angle $\theta_{1 / 2}$ of communication antenna is estimated with the following equation.

$$
\theta_{1 / 2}=70 \frac{\lambda}{D}
$$

Where $\lambda$ is wavelength of the received signal and $D$ is aperture of a parabolic antenna on the earth station.

Supposing the distance between the Sun and the earth station is $r_{s}$, and the apparent radius $\theta_{S}$ of the Sun satisfies the following equation.

Finally, the occurrence condition of sun outage is

$$
\theta_{S}=\frac{959.63}{r_{s}}
$$




$$
\theta \leq \theta_{1 / 2}+\theta_{S}
$$

With the criterion, whether a sun outage has occurred can be calculated. Furthermore, sun outage can be predicted on special period of time.

\subsection{Prediction Procedure of Sun Outage}

Combined with the above calculation model, calculation process of sun outage can be given to determine whether a sun outage occurs at special time. The procedure is as follows.

(1) Calculating half-power beam angle of the earth station antenna;

(2) Calculating azimuth angle and elevation angle of the communication satellite on the earth station at time $t$;

(3) Calculating azimuth angle and elevation angle of the Sun at time $t$, and the apparent radius $\theta_{S}$ of the Sun on the earth station;

(4) Using the spherical law of cosines to calculate the included angle $\theta$ of the Sun and the satellite;

(5) If the included angle $\theta$ satisfies $\theta \leq \theta_{1 / 2}+\theta_{S}$, sun outage will occur at time $t$; Otherwise, sun outage won't occur.

In this way, the occurring time of sun outage on the earth station will be obtained through cycling time. The algorithm flow chart of predicting sun outage is shown in Figure 2.

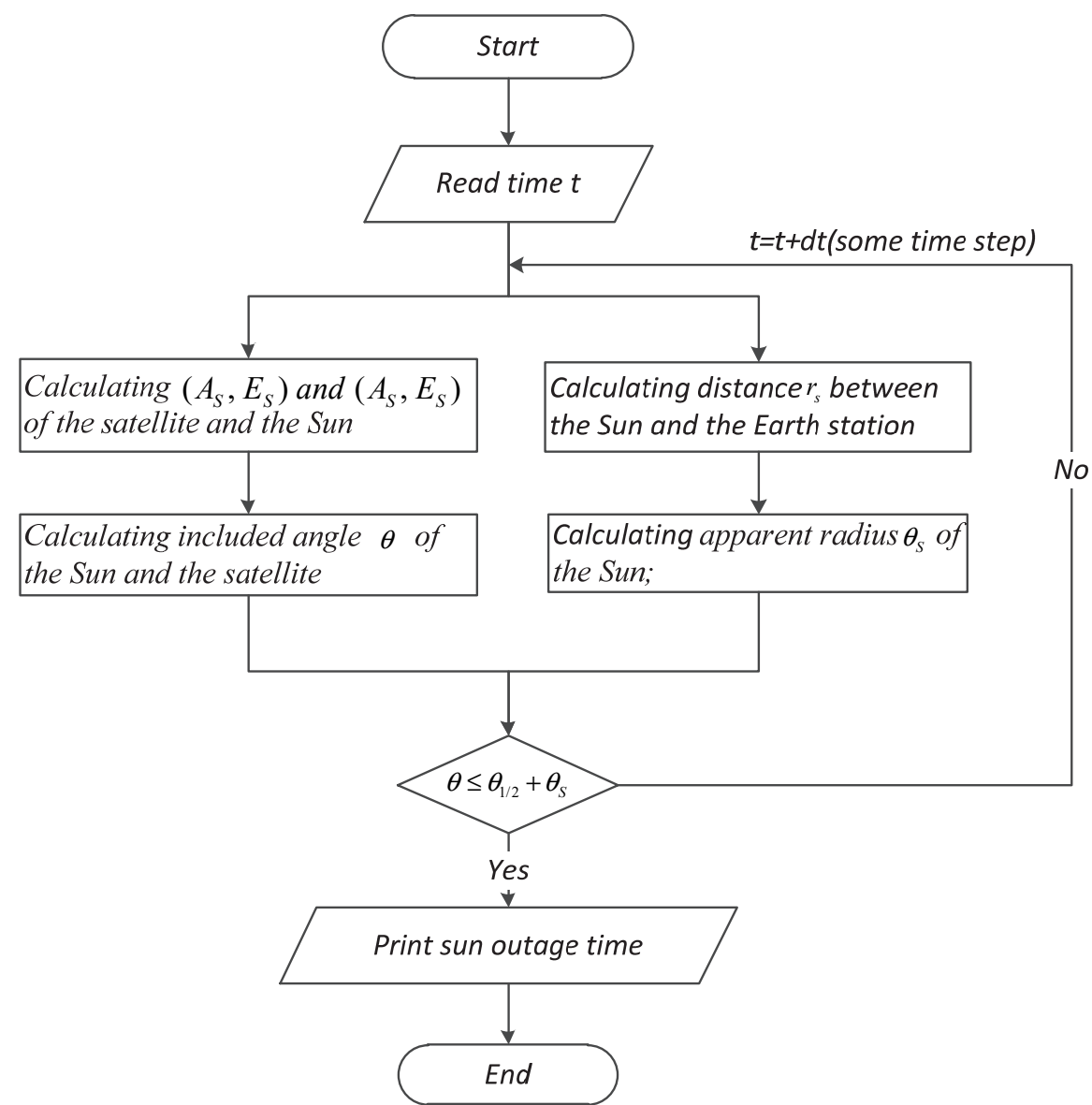

Figure 2. Flow chart of the prediction algorithm of sun outage

\section{Summary}

A sun outsage has a serious interference to satellite communication services in a special period of time. A novel model for predicting sun outage is proposed in this work. With this model, occurrence time of sun outage can be 
predicted with high precision on the earth station.

\section{References}

Guo, Y, Li, M. H., He, S. B., Xin, P., \& Tao, L. (2012). Analysis of the sun transit outage impact on the inter-satellite link of the navigation satellite. Lecture Notes in Electrical Engineering, 160, 133-143. https://doi.org/10.1007/978-3-642-29175-3_13

Lou, Y., Zhao, Y. W., Chen, C. Y., Tong, S. F., \& Han C. (2016). Analysis of sun outages influence on GEO to LEO communication. Lecture Notes in Electrical Engineering, 348, 1017-1025. https://doi.org/10.1007/978-81-322-2580-5_92

Pratt, T., Bostian, C. W., \& Allnutt, J. E. (2003). Satellite Communications (2nd ed.). John Wiley \& Sons, Inc.

Song, Y. J., Kim, K. S., Jin H., \& Lee, B. S. (2010). Prediction of communication outage period between satellite and earth station due to sun interference. Journal of Astronomy \& Space Sciences, 27(1), 31-42. https://doi.org/10.5140/JASS.2010.27.1.031

Tian, H. J., Zhao, Y. H., Cui, C. Z, \& Zheng, X. P. (2007). Design and implementation of China-VO ephemeris computing service based on web services. Astronomical Research \& Technology, 4(3), 283-287. https://doi.org/10.14005/ j.cnki.issn1672-7673.2007.03.009

\section{Notes}

Note 1.https://en.wikipedia.org/wiki/Sun_outage

Note 2. http://www.astro.com/

\section{Copyrights}

Copyright for this article is retained by the author(s), with first publication rights granted to the journal.

This is an open-access article distributed under the terms and conditions of the Creative Commons Attribution license (http://creativecommons.org/licenses/by/4.0/). 